\title{
Standardisierung von Allergenextrakten - eine Kunst für sich
}

\author{
Die Zeiten, da man Allergenextrakte noch durch ihren \\ Gehalt an Stickstoff definierte, sind endgültig vorbei. \\ Heute hat die Allergologie ein solides wissenschaftliches \\ Fundament; ihre molekularen Grundlagen sind transpa- \\ rent, das Fach ist, in den Worten U. Wahns, Berlin, \\ „high tech" geworden.
}

( ualitätssicherung bei Diagnose wie Therapie mit Allergenextrakten bedeutet in erster Linie eine Standardisierung der verwendeten Präparate, um Reproduzierbarkeit und Sicherheit zu gewährleisten. Daß diese Standardisierung ihre eigenen Tücken hat, erklärte kürzlich H. Løwenstein, Hørsholm/Dänemark: Hat man es dabei doch mit komplexen Proteinmischungen von Allergenmolekülen mit jeweils einer Vielzahl allergener Epitope zu tun. Und die Reaktionen der Patienten weisen eine große interindividuelle Variation auf sowohl hinsichtlich der Stärke der Reaktion, dem Protein, mit dem die IgE-Antikörper reagieren, sowie in bezug auf die jeweils relevanten Epitope.
Den optimalen Exktrakt für alle Patienten gibt es nicht, erklärte der Løwenstein; bei der Herstellung eines Allergenextrakts muß man sich an einem statistischen Durchschnitts-Allergiker orientieren.

\section{Rekombinante Allergene nicht der Königsweg}

In die Standardisierung eines optimalen Allergenextrakts müssen letztlich die Patientenreaktionen auf Zellwie Molekularebene ebenso wie die Konzentrationen der wichtigsten Einzelallergene mit eingehen. Dabei geht es darum, die Bandbreite möglicher Reaktionen des Durchschnittspatienten abzudecken. Das bedeutet, daß in einem optimalen Extrakt möglichst alle potentiellen Allergene vertreten

\section{Komplexe Vielfalt bei Allergenen wie Immunantworten}

Seit der Charakterisierung von über 100 Majorallergenen weiß man, daß diese in verschiedenen Isoformen existieren, welche voneinander oft nur in einer einzigen Aminosäure abweichen. Selbst in bezug auf die Bindung an verschiedene Isoformen gibt es individuelle Unterschiede zwischen den Patienten. Nachdem man fand, daß verschiedene Vertreter einer Baum- sorte jeweils nur eine beschränkte Auswahl der möglichen Bandbreite an Isoformen produzieren, ist es rein theoretisch - denkbar, daß ein Patient gegen die Pollen einer bestimmten Birke allergisch ist, gegen die einer anderen dagegen nicht. Letztlich dürfte es unmöglich sein, so Løwenstein, zwei Patienten zu finden, die identische allergische Antworten zeigen.
„Allergiker haben heute Anspruch auf molekular definierte Extrakte - diese sind ein gutes Stück Qualitätssicherung, die insbesondere Sicherheit bei der Therapiesteuerung mit sich bringt."

U. Wahn

sein müssen. Ein isoliertes Molekül herauszunehmen und $\mathrm{zu}$ reinigen, ist nach L $\varnothing$ wenstein „keine gute Idee“.

Aus diesem Grunde dürfte sich die Herstellung rekombinanter Allergene sicherlich nicht als Königsweg zu besseren Allergenpräparaten erweisen: Die gentechnische Herstellung eines definierten Moleküls ist zwar eine elegante Weise, eine gute Standardisierung zu gewährleisten; unter den Tisch fällt bei dieser Produktion jedoch die Vielzahl potentiell allergener Proteine und ihrer Isoformen. Ähnliche Bedenken führte Løwenstein gegen die T-Zell-Epitope an: Theoretisch ließe sich damit - durch Abwesenheit der B-Zell-Epitope - eine spezifische Allergentoleranz unter Ausschluß unerwünschter IgE-vermittelter Reaktionen induzieren; allerdings dürfte es angesichts der hohen Zahl solcher Epitope - jedes einzelne Peptidfragment eines Antigens ist ein potentielles Epitop - äußerst schwierig werden, die Gesamtheit der allergischen Antworten abzudecken. Zudem scheint die Rechnung mit der Senkung der Nebenwirkungsrate bisher nicht aufzugehen.

Die chemische Modifikation der Allergene induziert laut Løwenstein in erster Linie eine verstärkte IgGAntwort - was nicht zwangsläufig mit einer höheren Effektivität einhergeht.

(wpa)

4. Internationales Allergie-Symposium, Berlin, 20.-21.6.1997 (Veranstalter: Scherax Arzneimittel GmbH, Hamburg). 